\title{
Optimum design of bearing/seal parts for hydraulic equipment on mixed lubrication
}

\author{
Toshiharu KAZAMA and Atsushi YAMAGUCHI \\ Department of Mechanical Engineering and Materials Science, \\ Yokohama National University, \\ 156, Tokiwadai, Hodogaya-ku, Yokohama, 240, JAPAN
}

\begin{abstract}
Reasonable design criteria of the optimum size of bearing/seal parts of hydraulic equipment on mixed to fluid film lubrication are presented. We treat circular hydrostatic thrust bearings as the most fundamental model for the bearing/seal parts of hydraulic equipment. The basic equations considering metallic contact and eccentric loads are derived. The effects of the surface roughness, the ratio of pocket radius, and the radius of restrictors on the optimum size are clarified. The results are arranged by the concept of the ratio of hydrostatic balance. We show that the optimum criterion corresponding to the minimum power loss is given by the ratio of hydrostatic balance being close to unity.
\end{abstract}

\section{KEYWORDS}

Optimum design, Mixed lubrication, Hydrostatic bearings, Hydraulic equipment, Power loss

$$
\begin{aligned}
& \text { NOMENCLATURE } \\
& a= \text { ratio of pocket radius }=R_{1} / R_{2} \\
& A= \text { contact area } \\
& d_{\mathrm{e}}^{*}=\text { separation }=d_{\mathrm{e}} / \sigma^{*} \\
& E^{\prime}=\text { equivalent elastic modulus } \\
& f=\text { coefficient of friction } \\
& \bar{h}=\text { film thickness }=h / H \\
& h_{\mathrm{s}}=h / \sigma \\
& H=\text { representative film thickness } \\
& \bar{L}=\text { power loss }=L /\left[6 \mu \omega^{2} R_{2}{ }^{3}\left(R_{2} / H\right)^{2}\right]
\end{aligned}
$$

$$
\begin{aligned}
M & =\text { moment load } \\
N & =\text { speed of rotation } \\
p & =\text { pressure } \\
p_{\mathrm{r}}, p_{\mathrm{s}} & =\text { pocket and supply pressure } \\
Q & =\text { flow rate } \\
\bar{r}, \theta & =\text { coordinate }=r / R_{2}, \theta \\
R_{1}, R_{2} & =\text { pocket and outer radii } \\
S_{0} & =6 \mu \omega\left(R_{2} / H\right)^{2} / p_{\mathrm{s}} \\
T & =\text { frictional torque } \\
W & =\text { load; load carrying capacity }
\end{aligned}
$$


$\bar{x}_{\mathrm{w}}=$ eccentricity of load $=x_{\mathrm{w}} / R_{2}$

$\bar{\alpha}=$ tilting angle $=\alpha R_{2} / H$

$\delta=$ roughness

$\zeta=$ ratio of hydrostatic balance

$\mu=$ fluid viscosity

$\sigma=$ equivalent standard deviation of surface

$\tau=$ shear stress, time $=\omega t$

$\varphi=$ azimuth of maximum tilting angle

\section{Subscripts}

$a, f=$ asperity and fluid, respectively

1,2 = stationary and rotator, respectively

\section{INTRODUCTION}

Hydraulic equipment is operated under the conditions of high-pressure and wide-speed ranges. From industry applying hydraulic equipment for automation and energy saving, much lighter and smaller equipment is requested strongly. Furthermore, from the viewpoint of fire prevention and environmental protection, low viscous fluids, such as tap water, seawater, and high water content fluid (HWCF) are promoted to use as hydraulic fluids. On the other hand, the computer-aided design (CAD) of hydraulic equipment is also required. Therefore, it is necessary to clarify the optimum conditions and the design criteria of the bearing/seal parts of hydraulic equipment.

As to the optimum design criteria of bearings, there are some studies on thrust bearings [1], journal bearings [2], and so forth. These optimum conditions are based on the maximum stiffness in the fluid film lubrication range. Then, it is irrational to apply directly these criteria to the bearing/seal parts of hydraulic equipment.

With regard to the design criteria of the bearing/seal parts of hydraulic pumps and motors, there are some studies discussed the minimum power losses for slipper bearings on fluid film lubrication [3] and on mixed lubrication [4], and the maximum stiffness and the minimum leakage flow rate for pistons [5]. However, it is necessary to discuss much more generalized design criteria.

In this paper, we treat hydrostatic thrust bearings as the bearing/seal parts of hydraulic equipment on mixed to fluid film lubrication. We clarify numerically the optimum design criteria of the bearings for the surface roughness, the ratio of the pocket radius, and the radius of the restrictors.

\section{BASIC EQUATIONS}

Consider circular hydrostatic thrust bearings with a restrictor as shown in Fig. 1 .
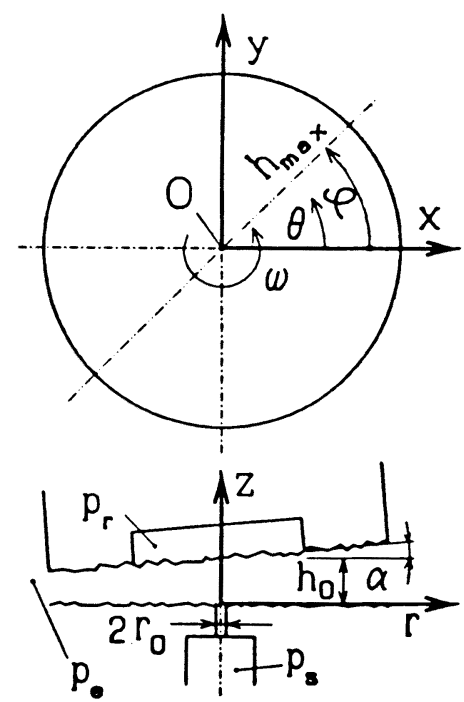

FIGURE 1 Schematic diagrams and coordinates 
The mixed lubrication model [6] is based on both of the asperities contacting model proposed by Greenwood and Williamson (the GW model) [7] and the mean flow model proposed by Patir and Cheng (the PC model) [8], and also included the effects of adsorption of lubricants, elastohydrodynamic lubrication(EHL), and cavitation due to asperities. It agrees well with many experimental results, and it is shown that the reliability of the model is reasonable.

The assumptions in this paper are: i) the roughness $\delta_{1}$ and $\delta_{2}$ are the normal distributions with the standard deviations $\sigma_{1}$ and $\sigma_{2}$, respectively, and $\delta=\delta_{1}+\delta_{2}, \sigma^{2}=\sigma_{1}^{2}+\sigma_{2}^{2}$; ii) the roughness is isotropic; iii) the configuration of an asperity close to a summit is a sphere; iv) the reference plane is rigid; $v$ ) the effects of heat and elastic deformation [9] are negligible, and vi) fluid is incompressible and its viscosity remains constant.

\section{Load carrying capacity}

For an area $\Delta A_{0}$, the load carrying capacities $\Delta W_{\mathrm{a}}, \Delta W_{\mathrm{ac}}$, and $\Delta W_{\mathrm{ap}}$ are

$$
\left.\begin{array}{rl}
\Delta W_{a}= & \Delta W_{a e}+\Delta W_{a p} \\
\Delta W_{a e}= & (2 / 3) E^{\prime}\left(\eta \beta^{\prime} \sigma^{*}\right)\left(\sigma^{*} / \beta\right)^{1 / 2} \\
& \times\left[F_{3 / 2}\left(d_{e}^{*}\right)-F_{3 / 2}\left(d_{e}^{*}+w_{p}^{*}\right)\right] \Delta A_{0} \\
\Delta W_{a p}= & \pi H_{a}\left(\eta \beta^{\prime} \sigma^{*}\right) F_{1}\left(d_{e}^{*}+w_{p}^{*}\right) \Delta A_{0}
\end{array}\right\}
$$

The load carrying capacity due to fluid is given by solving the Reynolds equation in the cylindrical coordinates $(\bar{r}, \theta, \bar{z})$ (see Appendix)

$$
\begin{aligned}
& \frac{1}{\bar{r}} \frac{\partial}{\partial \bar{r}}\left(\phi \bar{h}^{3} \bar{r} \frac{\partial p^{*}}{\partial \bar{r}}\right)+\frac{1}{\bar{r}^{2}} \frac{\partial}{\partial \theta}\left(\phi \vec{h}^{3} \frac{\partial p^{*}}{\partial \theta}\right) \\
& =6 \mu \omega\left(\frac{R_{2}}{H}\right)^{2}\left(\frac{\partial \bar{h}_{T}^{*}}{\partial \theta}-\frac{\sigma}{H} \frac{\partial \phi_{s}}{\partial \theta}+2 \frac{\partial \bar{h}_{T}^{*}}{\partial \tau}\right)
\end{aligned}
$$

where $\phi$ is a Couette shear factor and $\phi_{s}$ is a shear flow factor [8].

\section{Friction torque}

The friction torque due to asperities $T_{\mathrm{a}}$ is

$$
T_{a}=\iint_{A_{0}} R_{2} \bar{r} \Delta F_{a}
$$

where

$$
\begin{aligned}
& \begin{aligned}
\Delta F_{a} & =\xi \tau_{p}\left[\left(\tau_{e} d \tau_{p}\right) \Delta A_{e}+\Delta A_{p}\right] \\
& +(1-\xi) \tau_{a d} \Delta A_{r}
\end{aligned} \\
& \xi=\phi^{\prime} \exp \left[-\frac{\left(h_{a d} / a_{r}\right)^{m}}{\Delta A_{r} / \Delta A_{0}}\right]
\end{aligned}
$$

where $\phi^{\prime}=$ (coefficient of kinematic friction under boundary lubrication)/(that under dry friction) $=0.27$ (for steel), $a_{\mathrm{r}}=$ mean radius of asperities contacting $=\left[\beta^{\prime} \sigma^{*} F_{1}\left(d_{\mathrm{e}}^{*}\right) / F_{0}\left(d_{\mathrm{e}}^{*}\right)\right]^{1 / 2}, \quad h_{\mathrm{ad}}=\mathrm{EHL}$ film thickness of point contact, $\Delta A_{\mathrm{r}} / \Delta A_{0}=$ ratio of contacting area under dry friction $=\pi\left(\eta \beta^{\prime} \sigma^{*}\right) F_{1}\left(d_{\mathrm{e}}{ }^{*}\right)$, and $m=0.67+1.4 \sigma(\sigma: \mu \mathrm{m})$.

Following the PC model, the friction torque due to fluid acting on a rotator $T_{\mathrm{f} 2}$ is

$$
\begin{aligned}
T_{f 2}= & -\frac{\mu \omega R_{2}^{4}}{H} \int_{0}^{2 \pi} \int_{a}^{1} \frac{\bar{r}^{3}}{\bar{h}} \\
& \times\left[\phi+\left(1-2 \sigma_{2}^{2} / \sigma^{2}\right) \phi_{s}\right] \overline{d r} d \theta
\end{aligned}
$$


Flow rates and pocket pressure

In the case of an orifice restrictor, the flow rates through the restrictor $Q_{\text {in }}$ and into the bearing/seal parts $Q_{s}$ are

$$
\begin{aligned}
Q_{i n} & =\pi c_{0} r_{0}^{2} \sqrt{2\left(p_{s}-p_{r}\right) / \rho} \\
Q_{s}= & -a_{0}\left[\frac{p_{r}-p_{e}}{\log a}+\frac{3 \mu \omega}{a_{0}}\left(\frac{R_{2}}{H}\right)^{2} \frac{\partial c_{0}}{\partial \tau}\left(\frac{1-a^{2}}{\log a}+2 a^{2}\right)\right]_{(8)} \\
& -\bar{\alpha}^{2} a_{1}\left[\frac{A^{2}}{8} a^{2}\left(a^{2}-1\right)+\frac{C}{4} a^{2}\left(1-\frac{2 \log a}{a^{2}-1}\right)\right]
\end{aligned}
$$

The continuity equation of incompressible fluid is

$$
Q_{i n}=Q_{s}+\pi \omega a^{2} H R_{2}^{2} \partial \bar{h}_{T d}^{*} d \partial \tau
$$

The pocket pressure $p_{\mathrm{r}}$ is given by substituting Eqs.(7) and (8) into Eq.(9).

\section{Power losses, coefficient of friction, and ratio of hydrostatic balance}

The power loss $L$ and the coefficient of friction $f$ are defined by

$$
\begin{aligned}
& L=p_{s} Q_{s}+\omega\left(T_{a}-T_{f 2}\right) \\
& f=\left(T_{a}+T_{f 1}\right) /(-R W)
\end{aligned}
$$

where $R=$ mean radius $=\left(R_{1}+R_{2}\right) / 2$.

For the bearing/seal parts of hydraulic equipment, the loads are mainly supported by the hydrostatic pressure. Now, we introduce the ratio of the load to the maximum hydrostatic load carrying capacity $\zeta$ defined by

$$
\zeta=\frac{W}{\pi p_{s} R_{2}^{2}\left(1-a^{2}\right) /(2 \log a)}
$$

According to this definition, $\zeta<1$ implies the fluid film lubrication and $\zeta>1$ implies the mixed lubrication (in the case of metallic contact).

The examples of the numerical parameters are: $a=0.7, c_{0}=$ discharge coefficient $=0.6, E^{\prime}=200$ $\mathrm{GPa}, H_{\mathrm{a}}=1.75 \mathrm{GPa}, N=50 \mathrm{~s}^{-1}, p_{\mathrm{e}}=$ ambient pressure $=0 \mathrm{MPa}, p_{\mathrm{s}}=10 \mathrm{MPa}, r_{0}=$ radius of restrictor $=0.5$ $\mathrm{mm}, R_{2}=10 \mathrm{~mm}, \beta^{\prime}=0.05 \mathrm{~mm}, \quad \gamma=\mathrm{viscosity}-$ pressure coefficient $=22.8 \mathrm{GPa}^{-1}, \eta \beta^{\prime} \sigma^{*}=0.05$, $\mu=27.4 \mathrm{mPa} \cdot \mathrm{s}, \rho=$ density $=860 \mathrm{~kg} / \mathrm{m}^{3}, \sigma_{1}=\sigma_{2}=0.5$ $\mu \mathrm{m}, \tau_{\mathrm{ad}}=15 \mathrm{MPa}$.

\section{RESULTS AND DISCUSSIONS}

Figure 2 shows the effects of the ratio of hydrostatic balance $\zeta$ on the film thickness $h_{s}$, the ratio of real contact area to apparent area $A_{\mathrm{r}} / A_{0}$, the power losses due to leakage flow rate $\bar{L}_{\mathrm{Q}}$ and friction torque $\bar{L}_{\mathrm{T}}$, and the ratio of pocket pressure $p_{\mathrm{r}} p_{\mathrm{s}}$. $h_{\mathrm{s}}$ decreases with increasing $\zeta$. For $\zeta \propto 1, h_{\mathrm{s}}$ changes remarkably because the asperities begin to contact and support the loads. For hydrostatic bearings, we can recognize that this is the boundary between the fluid film and mixed lubrication ranges. For $\zeta<1, h_{\mathrm{s}}$ is very large because the bearing stiffness due to a restrictor is very small. $A_{\mathrm{r}} / A_{0}$ increases constantly with $\zeta$ in the mixed lubrication range. $p_{\mathrm{r}} / p_{\mathrm{s}}$ increases with $\zeta$ in the fluid film lubrication range and keeps nearly equal to unity in the mixed lubrication range. $\bar{L}$ is deeply dependent on $\bar{L}_{\mathrm{Q}}$ and $\overline{L_{\mathrm{T}}}$ for $\zeta<1$ and $\zeta>1$, respectively. 


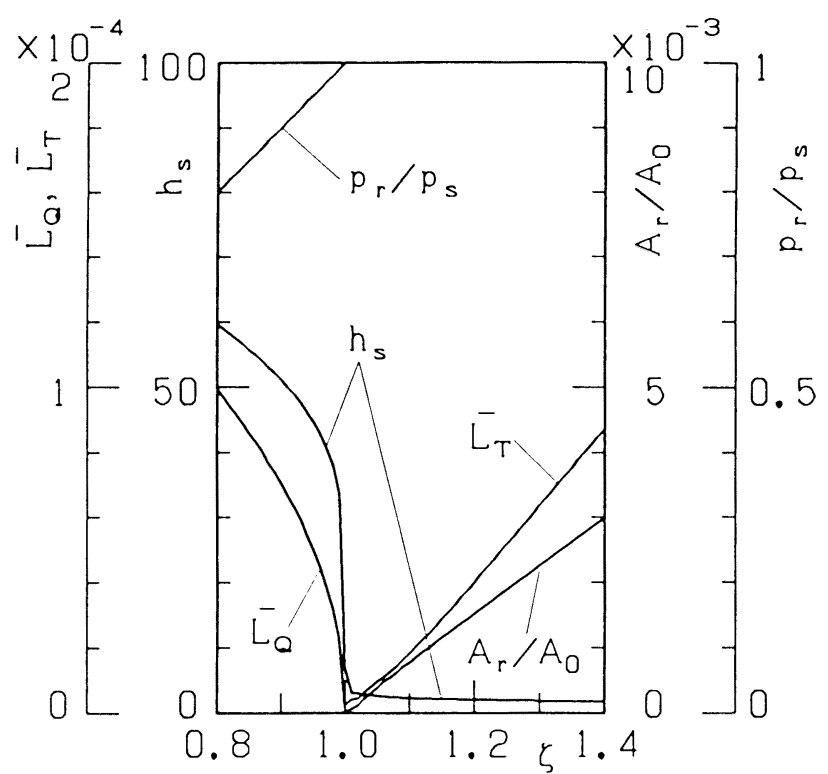

FIGURE 2 Effects of $\zeta$

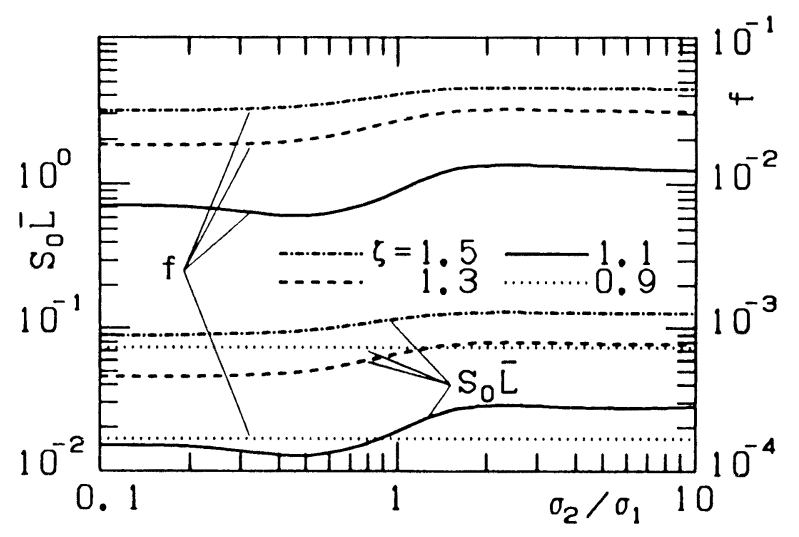

FIGURE 3 Effects of $\sigma_{2} / \sigma_{1}\left(\sigma_{1}=0.5 \mu \mathrm{m}\right)$

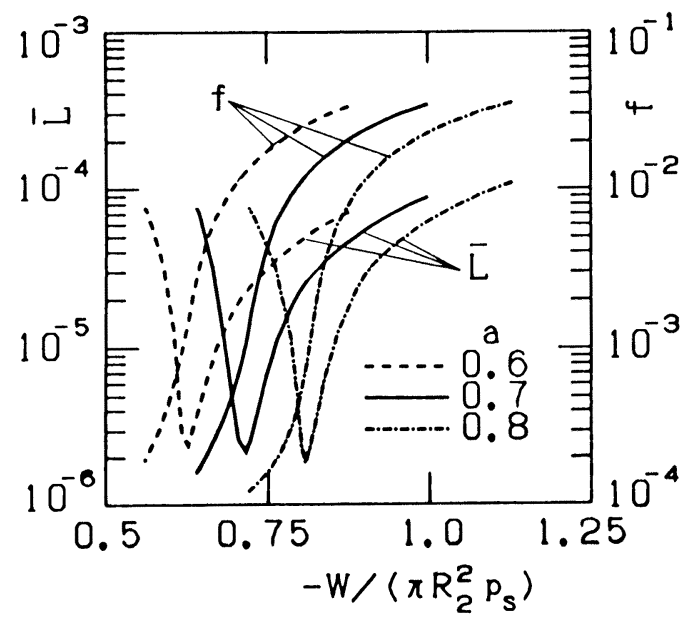

FIGURE 4 Effects of $a$

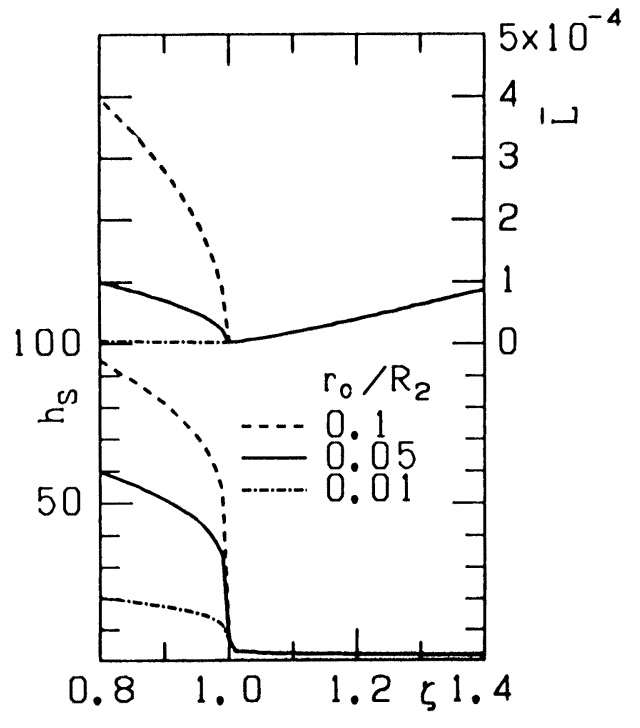

FIGURE 5 Effects of $r_{\mathrm{c}}$

Figure 3 shows the effects of roughness $\sigma_{2} / \sigma_{1}$ on the power loss $S_{0} \bar{L}$ and the coefficient of friction $f$. Any $\sigma_{2} / \sigma_{1}, S_{0} \bar{L}$ in the case of $\zeta=1.5$ is the largest and that of $\xi=1.1$ is the smallest. For $\zeta=1.1, \quad S_{0} \bar{L}$ and $f$ become the minimum at $\sigma_{2} / \sigma_{1} \sim 0.5$. Therefore, this is the optimum design criterion for surface roughness.

Figure 4 shows the effects of the ratio of pocket radius $a$ on $\bar{L}$ and $f . \bar{L}$ becomes the minimum at $\zeta \sim 1$ (see Fig. 2). For the same loads, the larger $a$, the smaller $\bar{L}$ and $f$ on $\zeta>1$.

Figure 5 shows the effects of the radius of restrictors $r_{\mathrm{c}}$ on $\bar{L}$ and $h_{0 \mathrm{~s}}$. The smaller $r_{\mathrm{c}}$, the smaller $\bar{L}$ on fluid film lubrication (no effects of $r_{\mathrm{c}}$ on mixed lubrication). However, considering the silting of the restrictors by contaminants in hydraulic fluids and the operating on mixed lubrication, we recommend to select the larger $r_{\mathrm{c}}$.

\section{CONCLUDING REMARKS}

Reasonable design criteria of the optimum size of bearing/seal parts of hydraulic equipment 
on mixed to fluid film lubrication are presented.

The minimum power loss is given in the ratio of hydrostatic balance being unity in all cases. The size of a restrictor does not influence power losses on mixed lubrication. Therefore, we recommend the optimum design criterion that the ratio of hydrostatic balance is set to slightly larger than unity.

\section{REFERENCES}

1. Shen, F. A., Optimum Stiffness of Externally Pressurized Thrust Bearings in Turbulent Regime, Trans. ASME, J. Lub. Technol. 1970, 457-465.

2. Rowe, W. B. and Koshal, D., A New Basis for the Optimization of Hybrid Journal Bearings, Wear, 64, 1980, 115-131.

3. Shute, N. A., Minimum Power Loss of Hydrostatic Slipper Bearings for Axial Piston Machines, Proc. Int. Conv. Lub. Wear, Inst. Mech. Eng., 1963, 3-14.

4. Iboshi, N., Characteristics of a Slipper Bearing for Swash Plate Type Axial Piston Pumps and Motors (3rd Report, Design Method for a Slipper with a Minimum Power Loss in Fluid Lubrication), Bull. Japan Soc. Mech. Eng., 29254, 1986, 2529-2538.

5. Laurenson, I. T., The Design of Self-Centring Seal-less Hydraulic Pistons, Proc. Instn. Mech. Eng., 199, 1985, 59-65.

6. Yamaguchi, A. and Matsuoka, H., A Mixed Lubrication Model Applicable to Bearing/Seal Parts of Hydraulic Equipment, Trans. ASME, J. Trib., 114, 1992, 116-121.

7. Greenwood, J. A. and Williamson, J. B. P., Contact of Normally Flat Surfaces, Proc. Roy. Soc. Lon., Ser. A., 295, 1966, 300-319.

8. Patir, N. and Cheng, H. S., An Average Flow Model for Determining Effects of Three-Dimensional Roughness on Partial Hydrodynamic Lubrication, Trans. ASME, J. Trib., 100, 1978, 12-17.

9. Kazama, T., Yamaguchi, A., and Shimizu, S., Thermoelastohydrodynamic Behavior of Hydrostatic Thrust Bearings (in Japanese), $J$. Japan Hydrau. Pneu. Soc., 21-4, 1990, 392-399.

\section{APPENDIX}

We apply the method of perturbation with the parameter $\bar{\alpha}$ to solve Eq.(1). The factors $\phi$, $\phi_{s}$, and the mean fluid film thickness (expectation) $h_{\mathrm{T}}{ }^{*}$ are approximated by the polynomial functions of the fourth order of $h_{s}$, that is

$$
\left.\begin{array}{rl}
\phi= & A_{0}+A_{1} h_{s}+A_{2} h_{s}^{2}+A_{3} h_{s}^{3}+A_{4} h_{s}^{4} \\
\phi_{s}= & \left(\sigma_{1}^{2}-\sigma_{2}^{2}\right) / \sigma^{2} \\
& \times\left(B_{0}+B_{1} h_{s}+B_{2} h_{s}^{2}+B_{3} h_{s}^{3}+B_{4} h_{s}^{4}\right) \\
h_{T}^{*}= & h+\sigma\left(C_{0}+C_{1} h_{s}+C_{2} h_{s}^{2}+C_{3} h_{s}^{3}+C_{4} h_{s}^{4}\right)
\end{array}\right\}
$$

The pressure $p^{*}=p_{0}{ }^{*}+\bar{\alpha} p_{1}{ }^{*}+\ldots$, where

$$
\begin{aligned}
p_{0}^{*} & =\frac{\log \bar{r}}{\log a} p_{r}+\left(1-\frac{\log \bar{r}}{\log a}\right) p_{e} \\
& +\frac{3 \mu \omega}{a_{0}}\left(\frac{R_{2}}{H}\right)^{2}\left[\frac{\left(1-a^{2}\right) \log \bar{r}}{\log a}+\bar{r}^{2}-1\right] \frac{\partial c_{0}}{\partial \tau} \\
p_{1}^{*} & =\frac{A}{8}\left[\bar{r}^{3}-\left(1+a^{2}\right) \bar{r}+\frac{a^{2}}{\bar{r}}\right] \cos (\varphi-\theta) \\
& +\frac{B}{8}\left[\bar{r}^{3}-\left(1+a^{2}\right) \bar{r}+\frac{a^{2}}{\bar{r}}\right] \sin (\varphi-\theta) \\
& +\frac{C}{2}\left(\bar{r} \log \bar{r}-\frac{a^{2} \log a}{a^{2}-1} \bar{r}+\frac{a^{2} \log a}{a^{2}-1} \frac{1}{\bar{r}}\right)
\end{aligned}
$$

The load carrying capacity $W_{\mathrm{f}}$, and moment load carrying capacities $M_{\mathrm{fx}}$ and $\boldsymbol{M}_{\mathrm{fy}}$ are

$$
\begin{aligned}
W_{f} & =R_{2}^{2} \int_{0}^{2 \pi} \int_{a}^{1} p^{*} \bar{r}\left(1-\frac{\Delta A_{r}}{\Delta A_{0}}\right) d \theta d \bar{r} \\
& +\pi R_{2}^{2}\left(a^{2} p_{r}-p_{e}\right)
\end{aligned}
$$

$$
\begin{aligned}
& M_{f x}=R_{2}^{3} \int_{0}^{2 \pi} \int_{a}^{1} p^{*} \bar{r}^{2} \sin \theta\left(1-\frac{\Delta A_{r}}{\Delta A_{0}}\right) \overline{d r} d \theta \\
& M_{f y}=-R_{2}^{3} \int_{0}^{2 \pi} \int_{a}^{1} p^{*} \bar{r}^{2} \cos \theta\left(1-\frac{\Delta A_{r}}{\Delta A_{0}}\right) d r d \theta
\end{aligned}
$$

